\title{
Lattice calculation of medium effects at short and long distances
}

\author{
P. Petreczky with O. Kaczmarek, F. Karsch, E. Laermann, S.Stickan, I. Wetzorke
} and F. Zantow

Fakultät für Physik, Universität Bielefeld

P.0. Box 10013133501 Bielefeld

We investigate medium effects in QCD like chromoelectric screening and quasi-particle mass generation by calculating the heavy quark potential as well as the temporal quark and gluon Coulomb gauge propagators in quenched approximation.

\section{INTRODUCTION}

The perturbative description of the Quark-Gluon Plasma (QGP) breaks down even at very high temperatures [1]. The reason for this is the generation of different length (time) scales at finite temperature. At high temperatures $\left(T \gg \Lambda_{Q C D}\right)$ the gauge coupling constant is small $g \ll 1$ and well separated length scales exist: $1 / T \ll 1 /(g T) \ll 1 /\left(g^{2} T\right)$. For such high temperatures the region of applicability of the perturbation theory is established. While for distances smaller than $1 / T$ the ordinary perturbation theory should be applicable, at distances smaller than $1 / g T$ the hard thermal loop resummed perturbation theory [2] should be applied. For distances larger than $1 / g^{2} T$, however, perturbation theory is no longer valid [1]. For interesting temperatures up to a few times $T_{c}$, however, $g \gtrsim 1$ and it is not clear up to which distances (times) the perturbation theory is applicable. At short distances $r \ll T^{-1}$ one expects that medium effects are not important. For such small distances one should also expect the ordinary perturbation theory to be viable, because at zero temperature perturbation theory works at short distances. Naturally the question arises: at what distances the medium effects become important and up to which distance perturbation theory is applicable? In the present work we try to answer these questions by studying the heavy quark potential and temporal quark and gluon propagators in Coulomb gauge.

\section{THE HEAVY QUARK POTENTIAL}

We consider the color averaged heavy quark potential defined by

$V(R, T)=-T \ln \left[\frac{<L(\vec{R}) L^{\dagger}(0)>}{|<L>|^{2}}\right]$.

Here $L(\vec{R})=\operatorname{Tr} \prod_{x_{0}=0}^{N_{\tau}-1} U_{0}\left(\vec{R}, x_{0}\right)$ is the Polyakov loop $\left(N_{\tau}\right.$ is the temporal extent of the lattice). So far most studies have concentrated on the long distance behavior of the heavy quark potential $(R T>1)$. For the physics of heavy quarkonia at finite temperature, however, it is important to know the potential for $R T<1$. In order to explore the short distance behavior of the heavy quark potential we performed simulations on $64^{3} \times 16$ 
lattices with the standard Wilson action at two different temperatures $T=1.5 T_{c}$ and $T=3 T_{c}$, as well as on $32^{3} \times 8$ lattices with a tree level Symanzik improved action for $T / T_{c}=1.05,1.14,1.23,1.50,3.0,6.0,12.0$. The color averaged potential $]^{-1}$ is related to the singlet $\left(V_{1}\right)$ and octet $\left(V_{8}\right)$ heavy quark potentials by the well-known formula [3]

$V(R, T)=-T \ln \left(\frac{1}{9} \exp \left(-\frac{V_{1}(R)}{T}\right)+\frac{8}{9} \exp \left(-\frac{V_{8}(R)}{T}\right)\right)$.

At very short distances $R T \ll 1$ one would expect that the singlet and octet potentials are given by their perturbative zero temperature expressions $V_{i}=c_{i} \alpha / R$, with $c_{1}=$ $-4 / 3$ for the singlet and $c_{8}=+1 / 6$ for the octet case. Thus for small distances one would expect $V(R, T) \sim 1 / R$. Expanding Eq. (2) up to $\alpha^{2}$ one recovers the well known perturbative result for the color averaged potential $V(R, T) / T=-\alpha^{2} /(3 R T)^{2}[3]$. We note, however, that the expansion of Eq. (2) couples the perturbative expansion to the high temperature expansion and therefore the above formula is obviously not valid at very short distances where $\left|V_{i} / T\right| \gg 1$. Motivated by the leading order perturbative result we define an effective coupling $\alpha_{e f f}^{2}(R, T)=-9 V(R, T) T[1 / R]^{-2}$. Here $[1 / R]$ denotes the lattice Coulomb potential. Our results on $\alpha_{e f f}(R, T)$ are summarized in Fig. 1a. One can see that there is a narrow region in $R T$ where $\alpha_{\text {eff }}$ is approximately constant as one would expect from the leading order perturbative result. We also note that in this region of $R T \alpha_{\text {eff }}(R, T)$ decreases with temperature as one would expect for the running coupling constant. For temperatures $T \leq 1.5 T_{c}$ the effective coupling seems to decrease at small distances $R T \lesssim 0.2$ which may imply that in this region the potential behaves like $1 / R$ according to the arguments given above. For higher temperatures the $1 / R$ behavior sets in at smaller distances because the coupling $\alpha$ is smaller and the high temperature expansion works well even at shorter distances. This probably is the reason that this behavior so far is not seen in our data for $T \geq 3 T_{c}$ which are restricted by the lattice spacing $a=0.0625 T^{-1}$ for $N_{\tau}=16$ and $0.125 T^{-1}$ for $N_{\tau}=8$. For distances $R T>0.3$ the effective coupling decreases with increasing $R T$ which implies the onset of screening. Thus we can distinguish three different regions of $R T$ which are characterized by qualitatively different behavior of $V(R, T)$ : the "true" short distance region where $V \sim 1 / R$, the intermediate perturbative region $V=-\alpha^{2} /(3 R T)^{2}$ and the "large" distance region where screening sets in. We note that for the lowest temperature $T=1.05 T_{c}$ the behavior of $V(R, T)$ is compatible with $1 / R^{2}$ for $R T>0.3$ which may imply that the effect of screening is very small. To quantify the effects of screening at higher temperatures it is useful to define an effective screening mass $\mu_{e f f}(R, T)=-\ln \left(-9 V(R, T)[1 / R]^{-2} / \alpha^{2}(T)\right) / R$ with $\alpha(T)=$ $\alpha_{\text {eff }}\left(R=0.125 T^{-1}, T\right)$. The behavior of $\mu_{e f f}(R, T)$ as function of $R$ is shown in Fig. $1 \mathrm{~b}$. As one can see from the figure the effective screening masses increase with increasing distance but up to distances $R T=1.5$ do not reach a plateau which can be identified with the true screening mass. In fact this behavior of the effective screening masses could be explained qualitatively in perturbation theory if one takes into account the momentum dependence of the gluon self energy $\Pi_{00}(k)$ [⿴囗十 . Note that the local screening masses in Fig. $1 \mathrm{~b}$ are always smaller than screening masses extracted from the large distance behavior of the heavy quark potential in [5] $\mu \sim 3 T$. They are also smaller than the perturbative

1 We stick here to the commonly used notion of a potential, although for $T>0$ one actually calculates the heavy quark free energy. 
a

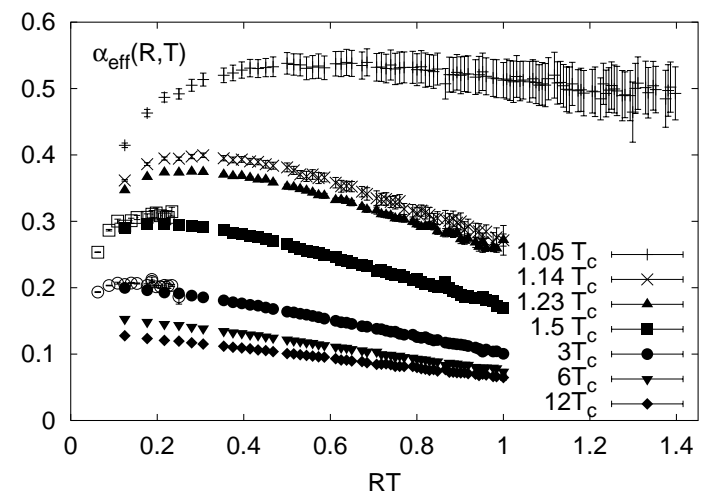

$\mathrm{b}$

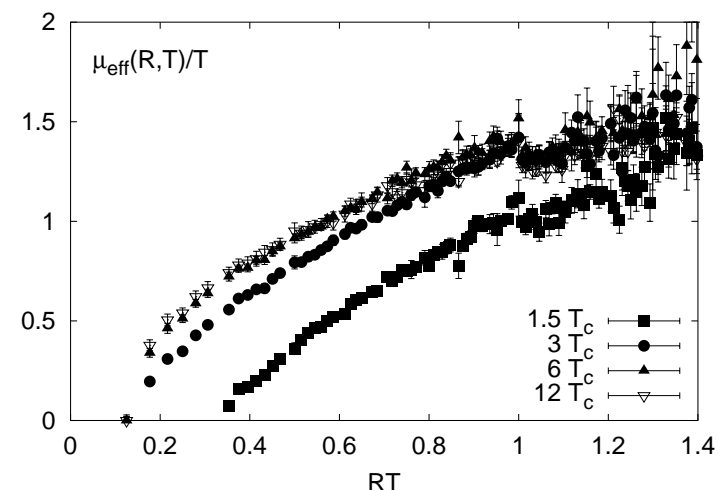

Figure 1. The effective coupling and the effective screening masses at different temperatures. The open symbols in the left figure are the results from simulations on a $64^{3} \times 16$ lattice with the Wilson action, others correspond to the data obtained on a $32^{3} \times 8$ lattice with an improved action.

Debye screening mass $g(T) T$ if $g$ is defined by $g(T)=\sqrt{4 \pi \alpha_{e f f}\left(R=0.125 T^{-1}, T\right)}$. We thus conclude that the color averaged heavy quark potential has a complex structure for interesting distances, $R T<1$, and temperatures a few times $T_{c}$.

\section{THE TEMPORAL QUARK AND GLUON PROPAGATORS}

The temporal quark and gluon propagators are related to properties of quasi-particles in the QGP. In fact, in hard thermal loop (HTL) approximation these propagators are dominated by quasi-particle poles. [6]. The quasi-particle picture of QGP finds application in resummed perturbative calculations of different thermodynamic quantities [7]. We have performed simulations in quenched QCD with Wilson fermions on a $64^{3} \times 16$ lattice at $T=3 T_{c}$. We have used the standard Wilson action for the gauge fields and an $O(a)$ improved fermion action (clover action). We have calculated the quark and gluon propagator $D_{i}(\tau, p)(i=q, g)$ in mixed $(\tau, p)$ representation ( here $p$ is the absolute value of the spatial momentum ).

The propagator in this mixed representation can be related to the spectral function by $D_{i}(\tau, p)=\int_{-\infty}^{+\infty} d \omega \rho(\omega, p) \exp (-\omega \tau) /(1 \pm \exp (-\omega \tau))$. Here $(i=q, g)$ and $+(-)$ correspond to the case of quarks (gluons). From the Monte-Carlo data for $D_{i}(\tau, p)$ we can extract the spectral functions $\rho(\omega, p)$ with the help of the Maximal Entropy Method (MEM) [8]. Since the quark and gluon propagator are gauge dependent quantities one has to fix a gauge. We have fixed the Coulomb gauge because in this gauge $\rho(\omega, p)$ is positive which is necessary for MEM. The peaks of the spectral function $\rho(\omega, p)$ define the dispersion relation $\omega(p)$ which is expected to be gauge invariant. Since the smallest non-zero momentum $p_{\min }=1.57 \mathrm{~T}$ is relatively large no detailed information about the properties of collective excitations like the longitudinal excitation in the gluon sector or the plasmino excitation in quark sector can be provided (these excitations exist only in the small momentum region [6]). Here we will not extract the spectral function but postpone that for future publications. Instead we compare our data for temporal quark and gluon propagators with the predictions of perturbation theory in HTL approximation. In Fig. 2a we show our results for the transverse gluon propagator $D_{g}^{T}(\tau, p)$ at momenta calculated on 40 gauge fixed configurations. We also show there the prediction of the HTL approximation using 
a

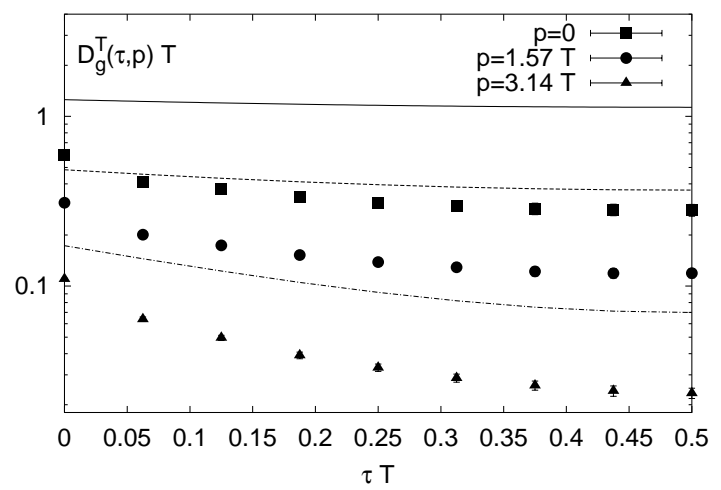

$\mathrm{b}$

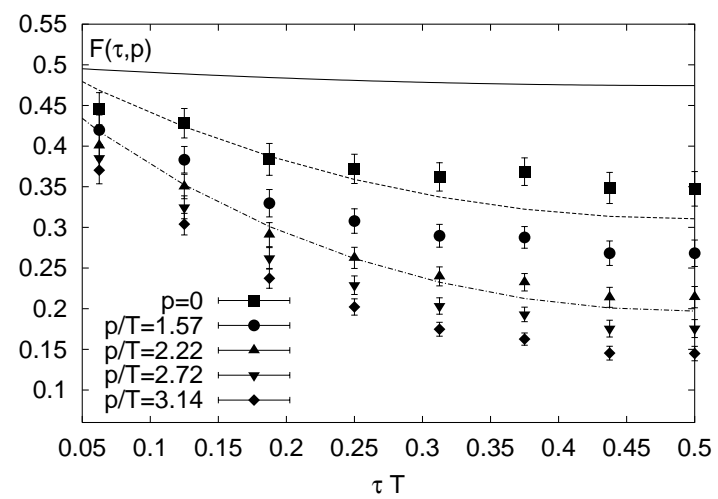

Figure 2. The transverse gluon propagator (a) and the quark propagators (b) for different momenta. The solid, dashed and dashed-dotted lines correspond to the prediction of perturbation theory in HTL approximation for momenta $p / T=0,1.57$ and 3.14 respectively.

a coupling constant $g \simeq 1.6$ suggested by the short distance behavior of the heavy-quark potential. As one can see from the figure the data disagree substantially with the HTL prediction even at momenta $p=3.14 T$. We note that corrections to the HTL approximation will not resolve this discrepancy since they lead to smaller $\omega(p)$ values shifting the propagator to values larger than the HTL result [9].

The quark propagator in mixed representation can be written as $D_{q}(\tau, p)=\gamma_{0} F(\tau, p)+$ $\vec{\gamma} \cdot \vec{n} G(\tau, p)+H(\tau, p)$ with $\vec{n}=\vec{p} / p$. In the chiral limit $\left(m_{q}=0\right) H(\tau, p)=0$. For the value of the hopping parameter $\kappa=0.1339$ (which corresponds to the quark mass close to the chiral limit) used in our simulation we have found $H(\tau, p)$ to be zero within the statistical accuracy reached in our simulations. In Fig. $2 \mathrm{~b}$ we show our results for $F(\tau, p)$ calculated on 40 configurations and compared with predictions of the HTL approximation. As in the case of the gluon propagator we find large deviations from the HTL predictions. The situation is similar for $G(\tau, p)$. We have found that there is no choice of $g$ which can provide agreement between lattice results and HTL. We thus conclude that at $T=3 T_{c}$ medium effects in temporal quark and gluon propagators are stronger than suggested by HTL perturbation theory.

\section{REFERENCES}

1. D.J. Gross et al, Rev. Mod. Phys. 53 (1981) 43

2. E. Braaten and R.D. Pisarski, Nucl. Phys. B337 (1990) 569

3. L.G. McLerran and B. Svetitsky, Phys. Rev. D24 (1981) 450

4. J.C. Gale and J. Kapusta, Phys. Lett. B198 (1987) 89

5. O. Kaczmarek et al, Phys.Rev. D62 (2000) 034021

6. M. Le Bellac, Thermal Field Theory (Cambridge University Press 1996)

7. J.O. Andersen et al, Phys. Rev. Lett.83 (1999) 2139; E. Iancu, these proceedings

8. M. Asakawa et al, hep-lat/0011040

9. H. Schulz, Nucl. Phys. B413 (1994) 353; A. Peshier et al, Ann. Phys. 266 (1998) 162 